\title{
The role of acceleration information in prism adaptation
}

\author{
ANDREA McCARTER \\ University of Georgia, Athens, Georgia 30602 \\ and \\ H. H. MIKAELIAN \\ University of New Brunswick, Fredericton, New Brunswick, Canada
}

\begin{abstract}
Two experiments were performed in which the acceleration component of limb movement information during prism exposure was manipulated, by controlling the trajectory and visibility of arm movement. When limb movements were confined to a lateral motion on a linear track, adaptation was evident when arm movement reversal at the end of the trajectory could be viewed (nonoccluded arm-movement reversal conditions). No adaptation occurred in the occluded arm-movement reversal condition. When movements were made on a curved track, adaptation was evident in both the nonoccluded and the occluded arm-movement reversal conditions. The results indicate that the acceleration component of reafferent stimulation may be critical in prism adaptation when no error information is available.
\end{abstract}

Numerous studies have dealt with the role of selfproduced movements in adaptation to a lateral displacement of the visual field (Held \& Bossom, 1961; Held \& Hein, 1958). Little attention, however, has been given to the type and geometry of self-generated movement necessary for adaptation to occur (Howard, 1968). Held's (Held \& Freedman, 1963) model asserts the need for reafferent stimulation, or visual feedback contingent on self-produced movement, for adaptation to prismatic displacement, a concept that is also applied to account for the development and maintenance of visually guided behavior. Inquiries into the types of reafferent stimulation that produce adaptation to prismatic displacement are essential for the elaboration of the reafferent model.

The general types of information contained in the visuospatial consequences of self-produced limb movements are: (a) the visual direction of the moved limb, (b) the speed of such motion, (c) change in the visual direction of the motion, and (d) change in the speed of such motion. The first two types of information are components of visual velocity, and the second two are components of visual acceleration. Visual velocity information is available to the subject when the arm moves in a fixed linear path at a constant speed. Acceleration information is introduced

This research was supported, in part, by a university-wide fellowship held by the first author at the University of Georgia. Mail reprint requests to $H$. Mikaelian, Department of Psychology, University of New Brunswick, Fredericton, New Brunswick, Canada. when either the speed of arm movement changes, as when the arm slows down, stops, and begins moving again, or the direction of arm movement changes, as on a curved path.

The first type of visual acceleration information, the one generated by change of speed of selfproduced arm motion, can be expressed by the following simple formula:

$$
\mathrm{A}=\frac{\Delta \mathrm{V}}{\Delta \mathrm{T}}=\frac{\mathrm{V}_{\mathrm{f}}-\mathrm{V}_{\mathrm{i}}}{\Delta \mathrm{T}},
$$

where $\mathrm{A}=$ acceleration, $\mathrm{V}=$ velocity, $\mathrm{T}=$ time, $\mathrm{V}_{\mathrm{f}}=$ final velocity, and $\mathrm{V}_{\mathrm{i}}=$ initial velocity. The second type of acceleration information, the one based on constant change of direction, is generated when the arm moves on a curvilinear path with a constant speed (illustrated in Figure 1, where velocity components are indicated by vectors). The length of each vector represents the speed of movement, which remains constant throughout the curved path. The indicated direction of movement is in relation to a polar coordinate system coincident with the central point of the circle formed by the arc (trajectory of arm movement). Acceleration in this case can be expressed by the following formulas:

$$
\begin{gathered}
\mathrm{A}=\mathrm{a}_{\mathrm{x}}+\mathrm{a}_{\mathrm{y}} \\
\mathrm{a}_{\mathrm{x}}=\frac{\Delta \mathrm{V}_{\mathrm{x}}}{\Delta \mathrm{T}}=\frac{\left(\mathrm{V}_{\mathrm{x}}\right)_{\mathrm{f}}-\left(\mathrm{V}_{\mathrm{x}}\right)_{\mathrm{i}}}{\Delta \mathrm{T}}
\end{gathered}
$$




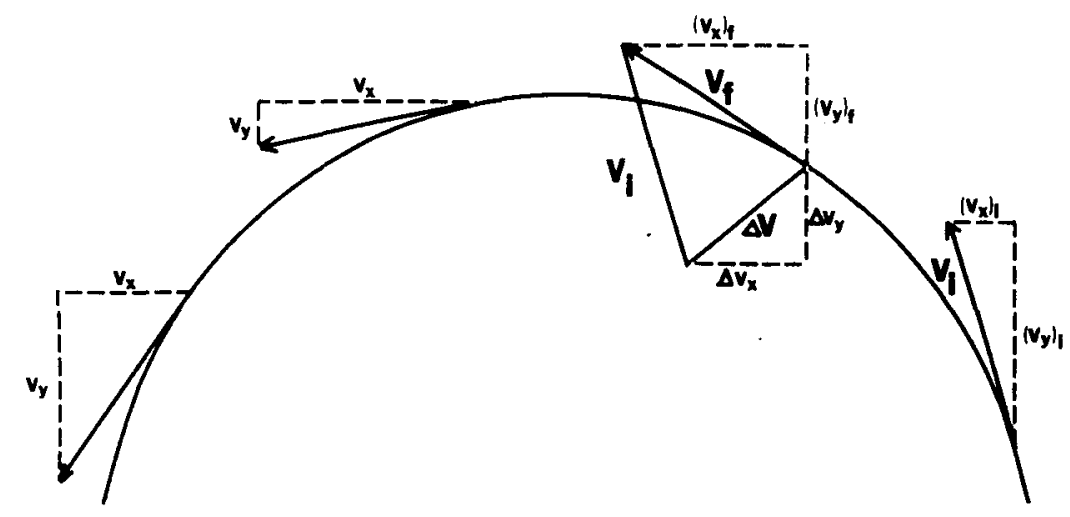

Figure 1. Acceleration on a curved path.

$$
a_{y}=\frac{\Delta V_{y}}{\Delta T}=\frac{\left(V_{y}\right)_{\mathrm{f}}-\left(V_{y}\right)_{i}}{\Delta T},
$$

where $\mathrm{x}=$ horizontal direction, $\mathrm{y}=$ vertical direction, $a_{x}=$ acceleration in a horizontal direction, and $a_{y}=$ acceleration in a vertical direction. The resulting acceleration on a curved path thus contains two components, acceleration in the horizontal and vertical directions of movement in relation to the centroid of the trajectory of arm movement.

Previous research has dealt with three motion variables in adaptation to prisms: the direction, speed, and change in the speed of movement. Freedman, Hall, and Rekosh (1965) investigated the influence of the direction of arm motion in the exposure and test responses. Comparing the effectiveness of sagittal arm motion and lateral motion through an arc, both under conditions of continuous viewing of the hand pointing at a target during exposure, they found no significant differences between the two movement conditions when similar exposure and test motion were used. When dissimilar exposure and test motions were used, more adaptation occurred when the exposure movements were sagittal and test movernents were in an arc than when exposure movements were in an arc and test movements were sagittal, Freedman et al. (1965) suggested that sagittal motion provides more relevant information for localization of the median plane than is provided by lateral motion through an arc. In all the conditions of their study, however, adaptation was significantly different from 0 . The availability of both velocity and acceleration information, as were defined earlier, in all the conditions in the Freedman et al. (1965) study may have been a contributing factor to this finding. Baily (1972) investigated the role of the speed of arm movements in adaptation, using terminal visual feedback during exposure (Cohen, 1967), in which only the final target pointing could be seen. He found that adaptation following fast arm movements generalized to both slow and fast movements. However, with slow arm movements during exposure, adaptation was evidenced only with slow test movements. In both of the above studies, response error information, based on the target pointing practice, was available during exposure.

Cohen $(1967,1973)$ has compared continuous visual feedback, during which the entire target reaching hand is visible, with terminal visual feedback, in which the stationary hand in its final target location is visible, with minimal deceleration visible to the subject. The amounts of adaptation generated in the two conditions were very similar, but the two conditions were distinguished by the occurrence of intermanual transfer in the terminal feedback condition, and its absence in the continuous feedback condition. Minimal information concerning acceleration based on change of speed, then, does not appear to diminish adaptation when error feedback from target pointing is available.

The exposure method used by Held and his collaborators (Held \& Gottlieb, 1958) is different from those used in the previous studies. Their subjects view limb movements along a more or less fixed, curvilinear path without view of any other part of the body or error feedback from target pointing. In Held's method, the limb remains continuously within the visual field and, since it moves along a curved path, these movements provide visual information regarding positive and negative acceleration of the arm, based on both change of direction and speed of arm movements. This paper reports two studies where visual acceleration information is manipulated by controlling the trajectory and geometry of arm movements. In Experiment 1, arm movements are designed to be confined to lateral trajectory on a linear track, thus minimizing acceleration information based on change of direction. In the first condi- 
tion of Experiment 1 (the nonoccluded armmovement reversal condition), the subject is permitted to view arm movement continuously. The arm moves back and forth along a linear path, each end of which is blocked to prevent the arm from moving outside the field of view. At each end of the track, the arm must slow down and stop, reverse direction, and regain speed again to reach the other end of the track, where the arm must again slow down and stop, and then repeat this procedure. Acceleration information that is generated by this condition is thus based on positive and negative acceleration (in terms of change in speed). In the second condition of Experiment 1 (the occluded arm-movement reversal condition), the subject's arm is permitted to move beyond the visual perimeter at each end of the track, thereby occluding view of the usual slowing down, stopping, and reversal of limb movement. View of arm movements is, then, limited to the middle portion of the track, where the arm moves at a more or less constant speed. In this condition, minimal acceleration information is available to the subject. If acceleration information is an essential component of reafferent stimulation, then adaptation to prismatic displacement should be evident in the nonoccluded arm-movement reversal condition (Condition 1), but not in the occluded arm-movement reversal condition (Condition 2 ).

In the second study, a curved track is used rather than a linear track, and the two conditions of occluded and nonoccluded arm movement are again compared. Acceleration information is, of course, available in both conditions, since the arm moves along a curved trajectory. In the occluded armmovement reversal condition, acceleration information is generated by the change of direction of arm movement (see Figure 1). Change of direction as well as change of speed generates acceleration information in the nonoccluded arm-reversal condition. Adaptation would, of course, be expected to occur in both the occluded and the nonoccluded armmovement reversal conditions, as acceleration information is generated in both.

The occluded and nonoccluded conditions in the present study can also be viewed as, respectively, discontinuous and continuous viewing conditions (as defined by Cohen, 1973). In the occluded armmovement reversal condition, view of the hand is alternated with occlusion of the hand with each armmovement reversal (when it goes outside the field of view). In the nonoccluded viewing condition, a continuous view of the hand throughout the exposure period is provided. As stated earlier, Cohen (1967) has reported that following discontinuous viewing of arm movements (in the form of terminal feedback), adaptation transfers intermanually; with continuous viewing, it does not. Taub and Goldberg
(1973) have argued that the continuous and discontinuous viewing conditions are equivalent to the massed vs. distributed practice conditions as defined in their "learned motor-skill" interpretation of prism adaptation. They contend that such a formulation is validated by the observation of larger initial aftereffect magnitude and intermanual transfer with distributed practice, as opposed to smaller aftereffects and no intermanual transfer with massed practice. If the Taub and Goldberg (1973) formulation is valid, then a higher aftereffect magnitude would be predicted for the occluded arm-movement reversal conditions in the present study (equivalent to their distributed practice conditions), than for the nonoccluded arm-movement reversal conditions (equivalent to their massed practice conditions). The predictions based on an interpretation of prism adaptation as a learned motor skill are thus directly contrary to the predictions based on the stipulation that adaptation is contingent upon acceleration information contained in self-produced arm movements.

\section{EXPERIMENT 1}

\section{Method}

Subjects. Fourteen right-handed undergraduates with no apparent visual defects participated in the experiment for course credit.

Apparatus. The testing apparatus consisted of an eye-hand coordination test box (Held \& Gottlieb, 1958) which allowed the subject to mark with his right hand the location of the virtual image of targets viewed in a mirror. Testing consisted of marking four targets 10 times each in a random order. The apparatus allowed right-eye monocular viewing of the test targets, and obscured both the subject's hand and his markings to prevent error feedback. The subjects' head was fixed with a biteboard rigidly attached to the apparatus during testing.

The exposure apparatus consisted of a slanted table top with a rectangular opening in which the prism goggles were mounted. The goggles allowed monocular right-eye viewing of arm movements through a 20 -diopter base-right prism. The subject's head rested on the goggles to reduce head movements. Arm movements were regulated by a sliding block which could be moved by the subject along a linear track mounted on a table. The table with the linear track was placed in front of the subject directly under the prism goggles. Stops at each end of the track were used to regulate the extent of arm traverse to allow continuous or discontinuous view of arm movement through the prism goggles. In the nonoccluded arm-movement reversal condition, the blocks were adjusted so that the length of the track was $42 \mathrm{~cm}$. In the occluded arm-movement reversal condition, the blocks were moved outwards an equal distance on each side of the track so that the length of the track was $71 \mathrm{~cm}$.

Procedure. Following preexposure testing of eye-hand coordination, the subject was instructed to view arm movements through the prism goggles as prescribed, i.e., moving the block on the track back and forth to each end of the track during each traverse, while viewing these arm movements with the head resting on the goggles. In the nonoccluded arm-movement reversal condition, arm movements were made on the short track, while in the occluded arm-movement reversal condition, the long track was 
used. Postexposure testing of eye-hand coordination (identical to preexposure) occurred at the end of the prescribed exposure period. The right eye was used throughout the experiment.

Prior to the experiment, the average single traverse time for each of the two viewing conditions was determined. In the occluded arm-reversal condition (arm extended outside the visual field) traverse time was $1.25 \mathrm{sec}$, and in the nonoccluded armreversal condition (arm remained within the visual field), traverse time was $.71 \mathrm{sec}$. A proportionate amount of time was added to the exposure period for the occluded arm-movement reversal condition, so that the total amount of time spent viewing the hand in both conditions was equated. Total exposure time for the occluded arm-movement reversal condition was $26.4 \mathrm{~min}$, while for the other condition it was $15 \mathrm{~min}$.

The same subjects participated in the two viewing conditions in two sessions separated by at least $24 \mathrm{~h}$. The order of conditions was balanced across the subjects.

\section{Results}

The results are shown in Table 1. Aftereffect measures, in degrees of visual angle, were determined by obtaining the mean differences between the centroids of the preexposure and postexposure target markings. Aftereffects in the adaptive direction are positive. In the nonoccluded arm-movement reversal condition, the mean aftereffect was $2.4^{\circ}$ of visual angle, reflecting $22 \%$ adaptation to the displacement. In the occluded arm-movement reversal condition, the mean aftereffect was about $.7^{\circ}$ of visual angle, indicating $6 \%$ adaptation. The aftereffect generated by the nonoccluded arm-movement reversal condition was significantly different from $0[\mathrm{t}(13)=7.34, \mathrm{p}<.001]$, whereas that produced by the occluded arm-movement reversal condition was not. The difference in adaptation between the two conditions was also significant $[\mathrm{t}(13)=2.4$, $\mathrm{p}<.05]$. Adaptation, then, was evident in the nonoccluded arm-movement reversal condition, but not in the occluded arm-movement reversal condition.

\section{EXPERIMENT 2}

\section{Method}

Subjects. Ten new right-handed undergraduate students with no apparent visual defects participated in the experiment for course credit.

Apparatus. The apparatus was identical to that used in the first experiment, except that the linear track was replaced with a curved track. The lengths of the curved track were $48.3 \mathrm{~cm}$ with a chord length of $42 \mathrm{~cm}$, for the nonoccluded arm-movement reversal condition, and $88.3 \mathrm{~cm}$ with a chord length of $63.5 \mathrm{~cm}$, for the occluded arm-movement reversal condition.

Procedure. The same procedure as in Experiment 1 was used. A proportionate amount of time was added to the occluded armmovement reversal condition, so that the total time spent viewing the hand in both conditions was equated. Exposure time for the occluded arm-movement reversal condition was $26 \mathrm{~min}$, and for the nonoccluded arm-movement reversal condition, $14 \mathrm{~min}$.

\section{Results}

The results are shown in Table 2 . In the occluded arm-movement reversal condition, the mean aftereffect was $3.2^{\circ}$ of visual angle, indicating $29 \%$ adaptation to the displacement. In the nonoccluded
Table 1

Changes (in Degrees of Visual Angle) in Eye-Hand Coordination Following Exposure to Arm Movement on a Linear Track With 20-Diopter Base-Right Prisms

\begin{tabular}{ccc}
\hline & \multicolumn{2}{c}{ Arm Movement Reversal } \\
\cline { 2 - 3 } Subject & Occluded & Nonoccluded \\
\hline 1 & 3.4 & 3.0 \\
2 & 2.9 & 4.5 \\
3 & 2.3 & 1.5 \\
4 & 2.9 & 1.1 \\
5 & -1.2 & 1.1 \\
6 & .5 & .1 \\
7 & -.5 & 2.7 \\
8 & 3.0 & 2.6 \\
9 & -4.4 & 3.6 \\
10 & -2.2 & 1.7 \\
11 & -1.3 & 3.2 \\
12 & -.3 & 3.4 \\
13 & 1.9 & 1.1 \\
14 & 2.2 & 1.8 \\
Mean & .66 & 2.4 \\
Percent & 6 & 22 \\
SD & 2.4 & 1.2 \\
\hline
\end{tabular}

arm-movement reversal condition, the mean aftereffect was $3.7^{\circ}$ of visual angle, indicating $34 \%$ adaptation to the displacement. The aftereffects were significantly reliable in both the occluded $[\mathrm{t}(9)=$ $2.4, \mathrm{p}<.05]$ and the nonoccluded $[\mathrm{t}(9)=4.85$, $\mathrm{p}<.001]$ conditions. No significant differences existed between the two conditions in Experiment 2 .

\section{DISCUSSION}

In the first experiment, adaptation was evident in the nonoccluded arm-movement reversal condition, but not in the occluded arm-movement reversal condition. The findings are thus in accord with the prediction that, when reafferent stimulation contains visual information concerning acceleration (in this case due to speed), adaptation occurs. However,

Table 2

Changes (in Degrees of Visual Angle) in Eye-Hand Coordination Following Exposure to Arm Movement on a Curved Track With 20-Diopter Base-Right Prisms

\begin{tabular}{ccc}
\hline & \multicolumn{2}{c}{ Arm Movement Reversal } \\
\cline { 2 - 3 } Subject & Occluded & Nonoccluded \\
\hline 1 & 2.7 & -.04 \\
2 & 2.5 & 2.1 \\
3 & 3.1 & 3.9 \\
4 & 11.4 & 5.0 \\
5 & 1.9 & 5.9 \\
6 & 5.1 & 6.3 \\
7 & 3.6 & 6.8 \\
8 & 6.8 & 4.9 \\
9 & -5.1 & 1.1 \\
10 & .4 & 1.3 \\
Mean & 3.2 & 3.7 \\
Percent & 29 & 34 \\
SD & 4 & 2.4 \\
\hline
\end{tabular}


when acceleration reafference is reduced to a minimum, as in the occluded arm-movement reversal condition, adaptation does not occur. Thus, with the Held type of exposure, reafference from selfproduced arm movements must include a visual acceleration component for adaptation to occur.

In the second experiment, adaptation was evident in both the nonoccluded and the occluded armmovement reversal conditions, as predicted, thereby suggesting that visual acceleration information based on constant change of direction is adequate for adaptation. In the occluded arm-movement reversal condition with a curved track, acceleration reafference from change in speed was minimized. That adaptation did occur indicates that a constant change in visual direction of movement is a sufficient source of relevant information. In the nonoccluded arm-movement reversal condition using a curved track visual feedback from both change of direction and change in speed were available, and adaptation was again evident.

These findings are not in accord with the predictions based on the "learned motor-skill" interpretation of adaptation phenomena. In the first experiment, the occluded arm-movement reversal viewing condition (the equivalent of distributed practice) was not sufficient for adaptation to occur, whereas the "motor-skill learning" approach would predict a higher magnitude of adaptation in this condition than in the nonoccluded arm-movement reversal condition. In the second experiment, both conditions generated equivalent adaptation, whereas a "learned motor-skill" interpretation would predict that more adaptation should have occurred in the occluded than in the nonoccluded arm-movement reversal condition.

The absence of significant adaptation in Experiment 1 (for the occluded arm-movement reversal condition with a linear track) could be attributable to the use of a lengthened hand-movement path, the presence of discontinuous reafferent stimulation, or the blocking of view of the stationary hand as well as the accelerating hand. The results in Experiment 2, however, argue against such an interpretation, as these three factors were again involved in the occluded arm-movement reversal condition on the curved track, without any significant decreases in adaptation.

It is possible that, in the same condition in Experiment 1 , acceleration information due to change of direction of arm movement was available to the subject, since the arm could be seen moving both to the left and to the right in between occlusions of the hand at the extremes of the trajectory. However, such a condition would provide minimal acceleration information, since only a single change of direction occurred during each stroke (in contrast to the con- tinually changing direction on a curved track), and each change of direction was separated by a relatively large time interval (it is more likely to assume that arm movements in each direction were mediated as discrete events, rather than as a form of acceleration). Such impoverished acceleration information might account for the small amount of residual adaptation $(6 \%)$ found in the occluded arm movement reversal condition in Experiment 1.

The results of the present study indicate that, when the Held-type exposure procedure is used (which reduces the typical response error feedback), the type of visual reafference available during eye-hand adaptation to a lateral displacement of the visual field is critical. They further suggest that, with this type of exposure, the acceleration component of reafference from arm movements facilitates adaptation to prismatic displacement, and that such acceleration information may be based on either change in speed or change in direction of movement. when information from both change in speed and change in direction are severely limited, no adaptation occurs.

The facilitating effect of acceleration information on prism adaptation found in the present study may be related to an enhanced responsivity of the visual system to changes in velocity compared to constantvelocity movement, as has been suggested by Rosenbaum (1975). Certainly, in other dimensions, such as brightness perception, the visual system is more responsive to changes in stimulation than to steady state stimulation (Hartline, 1938; Riggs, Ratliff, Cornsweet, \& Cornsweet, 1953). The prevalence of accelerated movement in comparison to constant velocity movement in most situations in which organisms encounter would make a sensitivity to acceleration information a useful characteristic (Rosenbaum, 1975).

\section{REFERENCES}

BaILy, John S. Adaptation to prisms: Do proprioceptive changes mediate adapted behavior with ballistic arm movements? Quarterly Journal of Experimental Psychology, 1972, 24, 8-20.

Conen. M. M. Continuous versus terminal visual feedback in prism aftereffects. Perceptual and Motor Skills, 1967, 24, 1295-1302.

COHEN. M. M. Visual feedback, distribution of practice, and intermanual transfer of prism aftereffect. Perceptual and Motor Skills, 1973, 37, 599-609.

Freedman, S. J., Hall, S. B., \& Rekosh, J. H. Effects on handeye coordination of two different arm motions during compensation for displaced vision. Perceptual and Motor Skills, 1965, 20. 1054-1056.

HARTLINE, H. K. The response of single optic nerve fibers of the vertebrate eye to illumination of the retina. American Journal of Physiology, 1938, 121, 400-415.

HELD, R.. \& Bossom, J. Neonatal deprivation and adult rearrangement: Complementary techniques for analyzing plastic sensorymotor coordinates. Joumal of Comparative and Physiological Psychology, 1961, 54, 33-37. 
Held, R.. \& Freedman. S. Plasticity in human sensorimotor con. trol. Science. 1\%63, 142, 455-462.

HELD. R.. \& GotTlieb. N. Technique for studying adaptation to disarranged hand-eye coordination. Perceptual and Motor Skills. 1958. 8. 83-86.

HELD. R.. \& HeIn, A. V. Adaptation of disarranged hand-eye coordination contingent upon re-afferent stimulation. Perceptual and Motor Skills, 1958, 8, 87-90.

HowarD. I. P. Displacing the optical array. In S. J. Freedman (Ed.). The neuropsychology of spatially oriented behavior. Homewood. 111: Dorsey Press. 1968.

Riggs, L. A.. Ratliff, F., Cornsweet, J. C., \& Cornsweet,
T. N. The disappearance of steadily fixated visual test objects. Journal of the Optical Society of America, 1953. 43, 495-501.

Rosenbaum, D. A. Perception and extrapolation of velocity and acceleration. Joumal of Experimental Psychology. 1975, 1. 395-403.

Taub, E., \& Goldberg, I. A. Prism adaptation: Control of intermanual transfer by distribution of practice. Science, 1973, 180. $755-757$.

(Received for publication February 7, 1977; revision accepted August 15, 1977.) 